\title{
A New Analytical Method for Solving General Riccati Equation
}

\author{
Yasar Pala, Mutlu Ozgur Ertas* \\ Engineering Faculty, Uludag University, Turkey
}

Copyright $\bigcirc 2017$ by authors, all rights reserved. Authors agree that this article remains permanently open access under the terms of the Creative Commons Attribution License 4.0 International License

\begin{abstract}
In this paper, the general Riccati equation is analytically solved by a new transformation. By the method developed, looking at the transformed equation, whether or not an explicit solution can be obtained is readily determined. Since the present method doesn't require a proper solution for the general solution, it is especially suitable for equations whose proper solutions cannot be seen at a first glance. Since the transformed second order linear equation obtained by the present transformation has the simplest form it can have, it is immediately seen whether or not the original equation can be solved analytically. The present method is exemplified by several examples.
\end{abstract}

Keywords Riccati Equation, Riccati, Ordinary Differential Equation, Nonlinear Differential Equation, Analytical Solution, Proper Solution

\section{Introduction}

The generalized Riccati equation is defined by

$$
\frac{d y}{d x}+P(x) y+Q(x) y^{2}-R(x)=0
$$

Here, $P(x), Q(x)$ and $R(x)$ are arbitrary functions of $x$. This equation is widely encountered in analytical mechanics, engineering and other fields. Therefore, depending on the functions $P(x), Q(x)$ and $R(x)$, several methods have been developed to solve various types of Riccati equations. [1-11]

If $(x)=0$, then the transformation $y=1 / z$ reduces Eq.(1) into a first order linear equation. For the case of $(x) \neq 0$, several methods are available in the literature [1-11]. The equation in this case is also known as Bernoulli equation.

The classical method for solving Riccati equation makes the transformation $\bar{y}=y_{0}+1 / y$, where $y_{0}$ is a known solution of the equation. However, it is not possible to see a proper solution to the equation at every time. Therefore, this method has a limited usage. Among other methods, Rao transformation and Allen-Stein transformation can be mentioned. The basic idea here is to bring the main equation into a separable form. $[1,7,8]$ However, the differentiability condition is a serious problem with these methods.

Harko, Lobo and Mak have investigated the Riccati Equation and developed a restricted analytical solution [2]. Since the method requires specific conditions among the coefficients in the equation, it cannot be considered a general method. Sugai transformed Riccati's equation into a second order differential equation by suggesting a new transformation. Since the transformed equation is more complicated and unsolvable in most cases, it is also not general and applicable [11]. Rao and Ukidave reduced Riccati's equation into a separable form under restricted condition [9]. It is of no importance in respect of engineering application. Siller also investigated a separability condition of the equation [10].

Integrability condition for the Riccati equation has been studied by Mak and Harko and a new method to generate analytical solutions of the Riccati equation was presented [5]. Mortici gives a new method of the variation of constants which leads directly to an equation with separable variables. This method also imposes several restrictions on the solution. Therefore, it cannot also be considered a general method [6].

When all methods are investigated, we observe that no method explains the question whether or not the analytical solution is obtained in explicit, implicit or power series form. In addition to finding the analytical solution to the problem for arbitrary values of $P(x), Q(x)$ and $R(x)$, the present method answers these important questions.

\subsection{Special Type Riccati Equation}

In order to solve Eq. (1), consider a new transformation in the form

$$
\bar{y}=f(x) e^{\int g(x) y(x) d x}
$$

where $(x), g(x)$ are functions to be determined in a convenient manner. We differentiate both sides of Eq. (2) twice :

$$
\bar{y}^{\prime}=\left(f^{\prime}+f g y\right) e^{\int g(x) y(x) d x}
$$

and 
$\bar{y}^{\prime \prime}=\left(f g y^{\prime}+2 f^{\prime} g y+f g^{\prime} y+f g^{2} y^{2}+f^{\prime \prime}\right) e^{\int g(x) y(x) d x}$

Eq. (4) can be written as

$$
y^{\prime}+\left[\frac{2 f^{\prime}}{f}+\frac{g^{\prime}}{g}\right] y+g y^{2}+\frac{f \prime \prime}{f g}=\frac{1}{f g} \bar{y}^{\prime \prime} e^{-\int g(x) y(x) d x}
$$

Recall that the left hand side of Eq. (5) has the form of Riccati equation. Comparing Eq. (1) and Eq. (5), we have

$$
\begin{gathered}
{\left[\frac{2 f^{\prime}}{f}+\frac{g^{\prime}}{g}\right]=P(x)} \\
g(x)=Q(x) \\
\frac{f^{\prime \prime}}{f g}=-R(x)
\end{gathered}
$$

If we wish to solve an equation of the form

$$
y^{\prime}+\left[\frac{2 f^{\prime}}{f}+\frac{g \prime}{g}\right] y+g y^{2}+\frac{f \prime \prime}{f g}=0
$$

then, by Eq.(5), in the first place, we can have

$$
\bar{y}^{\prime \prime}=0 \rightarrow \bar{y}=a x+b
$$

The functions $f$ and $g$ are to be obtained such that Eqs. (6) are satisfied. Now, using the inverse transformation from Eq. (2), we obtain

$$
y=\frac{1}{g} \frac{d}{d x}\left(\ln \frac{\bar{y}}{f}\right)
$$

We recall that, for some simple types of Riccati equations, the solution is obtained without having any difficulty in solving the second order equation (Eq. (8)). In other words, the solution is found without solving a complex second order differential equation with variable coefficients. The following four examples illustrate the method.

Example 1: We first try to solve the equation

$$
y^{\prime}+2 y+y^{2}+1=0
$$

whose solution can also be found by integration. Comparing Eq.(10) with Eq.(7) gives

$$
\begin{gathered}
{\left[\frac{2 f^{\prime}}{f}+\frac{g^{\prime}}{g}\right]=2} \\
g=1 \\
\frac{f^{\prime \prime}}{f g}=1
\end{gathered}
$$

Inserting $g=1$ into Eq. (11a) and solving the equation yields

$$
f=c e^{x}
$$

Eq. (11c) is automatically satisfied. Now, using Eq. (9), we have

$$
y=\frac{1}{g} \frac{d}{d x}\left(\ln \frac{\bar{y}}{f}\right)=\frac{1}{1} \frac{d}{d x}\left(\ln \frac{a x+b}{c e^{x}}\right)
$$

or

$$
y=\frac{1}{x+\bar{c}}-1, \quad \bar{c}=b / a
$$

It can be verified that Eq. (14) satisfies Eq. (10).

Example 2: We now solve the equation

$$
y^{\prime}+5 x y+y^{2}+\frac{25}{4} x^{2}+\frac{5}{2}=0
$$

Comparison of Eq. (15) with Eq. (7) yields

$$
\begin{gathered}
{\left[\frac{2 f^{\prime}}{f}+\frac{g^{\prime}}{g}\right]=5 x} \\
g=1 \\
\frac{f^{\prime \prime}}{f g}=\frac{25}{4} x^{2}+\frac{5}{2}
\end{gathered}
$$

The function $f$ can be obtained via Eq. (16a) and Eq. (16b)

$$
f=c e^{\frac{5}{4} x^{2}}
$$

Using Eq. (9), we find

$$
y=\frac{1}{g} \frac{d}{d x}\left(\ln \frac{\bar{y}}{f}\right)=\frac{1}{1} \frac{d}{d x}\left(\ln \frac{a x+b}{c e^{\frac{5}{4} x^{2}}}\right)
$$

or

$$
y=\frac{1}{(x+\bar{c})}-\frac{5}{2} x, \quad \bar{c}=b / a
$$

Again Eq. (19) satisfies Eq. (15).

Example 3: The equation that follows is required to be solved.

$$
y^{\prime}+2 x y+x^{2} y^{2}-\frac{1}{x^{2}}+\frac{2}{x^{4}}+1=0
$$

Comparison of Eq. (20) with Eq. (7) yields

$$
\begin{gathered}
{\left[\frac{2 f^{\prime}}{f}+\frac{g^{\prime}}{g}\right]=2 x} \\
g=x^{2} \\
\frac{f \prime \prime}{f g}=-\frac{1}{x^{2}}+\frac{2}{x^{4}}+1
\end{gathered}
$$

The function $f$ can readily be obtained

$$
f=c \cdot \frac{1}{x} \cdot e^{\frac{x^{2}}{2}}
$$

Using Eq. (9), we obtain

$$
y=\frac{1}{g} \frac{d}{d x}\left(\ln \frac{\bar{y}}{f}\right)=\frac{1}{x^{2}} \frac{d}{d x}\left(\ln \frac{a x+b}{c \cdot x^{-1} \cdot e^{\frac{x^{2}}{2}}}\right)
$$

or

$$
y=\frac{1}{x^{2}(x+\bar{c})}-\frac{1}{x}+\frac{1}{x^{3}} \quad \bar{c}=b / a
$$

In the examples above, the examples satisfying the condition $\bar{R}(x)=f^{\prime \prime} / f g$ automatically have been chosen. Otherwise, the unknown function $f(x)$ must be found such that the equations

$$
\begin{gathered}
\bar{P}(x)=\left[\frac{2 f^{\prime}}{f}+\frac{g^{\prime}}{g}\right] \\
\bar{Q}(x)=g \\
\bar{R}(x)=\frac{f^{\prime \prime}}{f g}
\end{gathered}
$$

must be simultaneously satisfied. However, it is not always possible to obtain a unique $f(x)$ that satisfies both Eq. (25a) and Eq. (25c). To show this case, we finally consider 
following example in this section.

Example 4: We try to solve the equation

$$
y^{\prime}+\alpha x^{2} y+\beta x y^{2}+\bar{R}(x)=0
$$

Using Eq. (25a) and Eq. (25b), we can write

$$
\begin{gathered}
{\left[\frac{2 f^{\prime}}{f}+\frac{g^{\prime}}{g}\right]=\alpha x^{2}} \\
g=\beta x
\end{gathered}
$$

The function $f$ can be obtained as

$$
f=c \cdot x^{-\frac{1}{2}} \cdot e^{\frac{\alpha x^{3}}{6}}
$$

We then have

$$
\bar{R}(x)=\frac{f^{\prime \prime}}{f g}=\frac{\alpha^{2}}{4 \beta} x^{3}+\frac{3}{4 \beta} x^{-3}+\frac{\alpha}{2 \beta}
$$

Thus the present method gives a solution as long as the condition given in Eq. (29) is satisfied. The new form of Eq. (24) reads

$$
y^{\prime}+\alpha x^{2} y+\beta x y^{2}+\frac{\alpha^{2}}{4 \beta} x^{3}+\frac{3}{4 \beta} x^{-3}+\frac{\alpha}{2 \beta}=0
$$

The solution can be obtained to be

$$
y=\frac{1}{g} \frac{d}{d x}\left(\ln \frac{\bar{y}}{f}\right)=\frac{1}{\beta x} \frac{d}{d x}\left(\ln \frac{a x+b}{c \cdot x^{-\frac{1}{2}} \cdot e^{\frac{\alpha x^{3}}{6}}}\right)
$$

or

$$
y=\frac{1}{\beta x(x+\bar{c})}-\frac{\alpha x}{2 \beta}+\frac{1}{2 \beta x^{2}} \quad \bar{c}=b / a
$$

\subsection{The Riccati Equation of General Type}

The transformation in Eq. (2) prevents the free choice of the term $Q(x)$ in Eq. (1). So, the method can be valid only for special type Riccati equations. Now, we wish to remove this restriction to have the analytical solution of general type Riccati equation involving arbitrary $P(x), Q(x)$ and $R(x)$. Again, we consider the same transformation

$$
\bar{y}=f(x) e^{\int g(x) y(x) d x}
$$

Differentiating Eq. (33) twice yields

$$
\bar{y}^{\prime}=\left(f^{\prime}+f g y\right) e^{\int g(x) y(x) d x}
$$

and

$$
\bar{y}^{\prime \prime}=\left(f g y^{\prime}+2 f^{\prime} g y+f g^{\prime} y+f g^{2} y^{2}+f^{\prime \prime}\right) e^{\int g(x) y(x) d x}
$$

Eq. (35) can be written as

$$
y^{\prime}+\left[\frac{2 f^{\prime}}{f}+\frac{g^{\prime}}{g}\right] y+g y^{2}+\frac{f \prime \prime}{f g}=\frac{1}{f g} \bar{y}^{\prime \prime} e^{-\int g(x) y(x) d x}
$$

In order to remove the restriction in the section 1.1, we now assume that we seek the solution of the equation

$$
y^{\prime}+\left[\frac{2 f^{\prime}}{f}+\frac{g^{\prime}}{g}\right] y+g y^{2}+\frac{f \prime \prime}{f g}=S(x)
$$

where $S(x)$ is a function to be determined. Thus, Eq. (36) gives

$$
\bar{y}^{\prime \prime}=f \cdot g \cdot S(x) \cdot e^{\int g(x) y(x) d x}
$$

However, by Eq. (33), we can also write

$$
\bar{y}^{\prime \prime}=g S(x)(\bar{y})
$$

Replacing $\bar{y}$ by $u(x)$, we obtain

$$
u^{\prime \prime}-g S u=0
$$

Eq. (40) is a linear second order ordinary differential equation whose general solution is generally obtained in power series. However, if $g S$ is a constant or something that leads to the analytical solution of Eq.(40), then the explicit form of $u$ or $y$ can always be obtained.

Now, an attention should be paid to Eq. (40). According to this result, we are led to conclude that one of the main advantages of the transformation given by Eq. (33) is that it converts Riccati equation directly into the linear form of Eq. (40). Eq. (40) has the simplest form of a second order differential equation. This form also provides us the knowledge of whether we can obtain the explicit solution of Eq. (37) or not. After Eq. (40) is solved via classical methods, the inverse transformation yields the solution as

$$
y=\frac{1}{g(x)} \frac{d}{d x}\left(\ln \frac{u(x)}{f(x)}\right)
$$

Since Eq. (41) doesn't involve any expression to be integrated, there exists no difficulty in obtaining the explicit form of $y(x)$.

Example 5: As a first example in this section, we solve the equation

$$
y^{\prime}+2 x y-y^{2}-\left(1+x^{2}\right)=0
$$

Comparing Eq. (42) and Eq. (37), we have

$$
\begin{gathered}
g=-1 \\
{\left[\frac{2 f^{\prime}}{f}+\frac{g^{\prime}}{g}\right]=2 x} \\
\frac{f^{\prime \prime}}{f g}=-\left(1+x^{2}\right), \quad S(x)=0
\end{gathered}
$$

Solving Eq. (43a) and Eq. (43b) gives $f=c e^{x^{2} / 2}$ ( $c=$ constant) and we can see immediately that $f^{\prime \prime} / f g$ directly equals to $R(x)$. Hence, $S(x)$ can be taken as zero. This means that $\bar{y}^{\prime \prime}=0$. The solution of $\bar{y}$ is given by $\bar{y}=a x+b$. Substituting this result into the inverse transformation, after some operations, we obtain

$$
y=x-\frac{1}{x+\bar{c}} \quad(\bar{c}=\text { constant })
$$

It can be checked that Eq. (44) satisfies Eq. (42).

Example 6: We seek the solution of the equation

$$
y^{\prime}+8 x y+4 y^{2}+4 x^{2}-3=0
$$

Comparing Eq. (37) and Eq. (45), we have

$$
\begin{gathered}
g=4 \\
{\left[\frac{2 f^{\prime}}{f}+\frac{g^{\prime}}{g}\right]=8 x}
\end{gathered}
$$

Solving Eq. (46a) and Eq. (46b) gives $f=c e^{2 x^{2}}$ ( $c=$ constant). The other condition yields

$$
\frac{f \prime \prime}{f g}=4 x^{2}+1, \quad S(x)=4
$$


The transformed equation in this case becomes

$$
u^{\prime \prime}-16 u=0
$$

The characteristic equation of Eq. (48) is

$$
m^{2}-16=0
$$

whose roots are $m_{1}=4$ and $m_{2}=-4$. Thus, the solution of Eq. (48) has the form

$$
u=c_{1} e^{4 x}+c_{2} e^{-4 x}
$$

Using Eq. (41), we obtain $y(x)$ as

$$
\begin{gathered}
y=\frac{1}{g(x)} \frac{d}{d x}\left(\ln \frac{u(x)}{f(x)}\right)=\frac{1}{4} \frac{d}{d x}\left(\ln \frac{c_{1} e^{4 x}+c_{2} e^{-4 x}}{c e^{2 x^{2}}}\right) \\
y=\frac{c_{1} e^{4 x}-c_{2} e^{-4 x}}{c_{1} e^{4 x}+c_{2} e^{-4 x}}-x
\end{gathered}
$$

It can be verified that

$$
y=\frac{e^{8 x}-\bar{c}}{e^{8 x}+\bar{c}}-x, \quad\left(\bar{c}=c_{2} / c_{1}\right)
$$

Note that when we take $c_{1} e^{4 x}$ as the solution of $u$, then we obtain the proper solution $y_{p}=1-x$. In addition, if we take $c_{2} e^{-4 x}$ only, then we find the other proper solution of Eq. (44) as $y_{p}=-1-x$. Indeed, we can verify that these are proper solutions of Eq. (45). Thus, the method presented also gives the proper solutions of Riccati Equation. Then, when desired, one can use the famous transformation $y=\bar{S}(x)+1 / x$ to find the general solution. Here, $\bar{S}(x)$ is a proper solution.

Example 7: We now try to solve the equation

$$
y^{\prime}+\left(4-\frac{1}{x}\right) y+\frac{1}{x} y^{2}-x^{2}+4 x=0
$$

We recall that a proper solution of Eq. (54) is not readily seen. Therefore, the well-known classical method cannot be used. It is obvious that the following equations are valid:

$$
\begin{gathered}
g=\frac{1}{x} \\
{\left[\frac{2 f^{\prime}}{f}+\frac{g^{\prime}}{g}\right]=4-\frac{1}{x}}
\end{gathered}
$$

Solving Eq. (55a) and Eq. (55b) gives $f=c e^{2 x}$ ( $c=$ constant). Now, noting that

$$
\frac{f^{\prime \prime}}{f g}=4 x
$$

from Eq.(37), we obtain $S(x)$ as

$$
\frac{f^{\prime \prime}}{f g}-S(x)=-x^{2}+4 x \Rightarrow 4 x-S(x)=-x^{2}+4 x \Rightarrow S(x)=x^{2}
$$

Hence, Eq. (40) reads

$$
u^{\prime \prime}-x u=0
$$

Eq. (58) is Airy equation and its solution is given by

$$
\bar{y}=u=c_{1} A i(x)+c_{2} B i(x)
$$

Here, the functions $\operatorname{Airy} A i(x)$ and $\operatorname{AiryBi}(x)$ are given as

$$
A i(x)=1+\sum_{n=1}^{\infty} \frac{x^{3 n}}{2.3 .5 \cdot 6 \ldots .(3 n-3) \cdot(3 n-1) \cdot 3 n}, \quad B i(x)=x+\sum_{n=1}^{\infty} \frac{x^{3 n+1}}{3.4 \cdot 6 \cdot 7 \ldots(3 n-2) \cdot 3 n \cdot(3 n+1)}
$$

Their derivatives are given by

$$
(A i(x))^{\prime}=\sum_{n=1}^{\infty} \frac{x^{3 n-1}}{2.3 .5 .6 \ldots . .(3 n-3) \cdot(3 n-1)}, \quad(B i(x))^{\prime}=1+\sum_{n=1}^{\infty} \frac{x^{3 n}}{3.4 .5 .6 .7 \ldots(3 n-2) \cdot 3 n}
$$

Hence, using the inverse transformation, we obtain

$$
y=x\left(\frac{(A i(x))^{\prime}+c \cdot(B i(x))^{\prime}}{A i(x)+c \cdot B i(x)}-2\right)
$$

where $c$ is a constat. 
Example 8: We try to solve the equation which has been studied by Mortici [6]

$$
y^{\prime}-\frac{\beta}{x} y-\alpha y^{2}-\frac{\gamma}{x^{2}}=0
$$

Comparing Eq. (37) and Eq. (63), we have

$$
\begin{gathered}
g=-\alpha \\
{\left[\frac{2 f^{\prime}}{f}+\frac{g^{\prime}}{g}\right]=-\frac{\beta}{x}}
\end{gathered}
$$

Solving Eq. (64a) and Eq. (64b) gives $f=c x^{-\beta / 2}$. ( $c=$ constant). Noting that

$$
\frac{f^{\prime \prime}}{f g}=\frac{-\left(\beta^{2}+2 \beta\right)}{4 \alpha} \cdot \frac{1}{x^{2}}
$$

from Eq. (37), we obtain $S(x)$ as

$$
\begin{gathered}
\frac{f^{\prime \prime}}{f g}-S(x)=-\frac{\gamma}{2} \Rightarrow \frac{-\left(\beta^{2}+2 \beta\right)}{4 \alpha} \cdot \frac{1}{x^{2}}-S(x)=-\frac{\gamma}{2} \\
S(x)=\left(\gamma-\frac{\left(\beta^{2}+2 \beta\right)}{4 \alpha}\right) \cdot \frac{1}{x^{2}}
\end{gathered}
$$

We have the transformed equation below via Eq. (40):

$$
\begin{gathered}
u^{\prime \prime}-(-\alpha)\left(\gamma-\frac{\left(\beta^{2}+2 \beta\right)}{4 \alpha}\right) \frac{1}{x^{2}} u=0 \\
u^{\prime \prime}+\left(\frac{4 \gamma \alpha-\left(\beta^{2}+2 \beta\right)}{4}\right) \frac{1}{x^{2}} u=0
\end{gathered}
$$

After this point, the following cases can be considered.

Case 1: If we assume $4 \gamma \alpha=\left(\beta^{2}+2 \beta\right)$, the transformed equation in this case becomes

$$
u^{\prime \prime}=0
$$

The form of $u$ must be $u=a x+b$. Hence, Eq. (41) gives

$$
y=\frac{1}{g} \frac{d}{d x}\left(\ln \frac{u}{f}\right)=-\frac{1}{\alpha} \frac{d}{d x}\left(\ln \frac{a x+b}{c x^{-\beta / 2}}\right)
$$

or

$$
y=\frac{-1}{\alpha(x+\bar{c})}-\frac{\beta}{2 \alpha x} \quad \bar{c}=b / a
$$

Case 2: If we assume $4 \gamma \alpha \neq\left(\beta^{2}+2 \beta\right)$ as a general case, then we can put $k=\frac{4 \gamma \alpha-\left(\beta^{2}+2 \beta\right)}{4}=$ const. the transformed equation in this case becomes

$$
u^{\prime \prime}+\frac{k}{x^{2}} u=0
$$

This is an Euler-Cauchy Equation which has the general form.

$$
u^{\prime \prime}+\frac{A}{x} u^{\prime}+\frac{B}{x^{2}} u=0
$$

This equation can be transformed into the linear form as

$$
Y^{\prime \prime}+(A-1) Y^{\prime}+B Y=0, \quad(A=0, B=k)
$$

The characteristic equation of Eq. (74) is

$$
m^{2}-m+k=0
$$

whose roots are $m_{1}$ and $m_{2} \cdot m_{1}$ and $m_{2}$ can be real, complex or repeated. The form of solution depends on the types of the roots.
Case a: If $(1-4 k)>0, \quad m_{1} \neq m_{2}$ and $m_{1}, m_{2} \in \mathbb{R}$. $m_{1,2}=\frac{1 \pm \sqrt{1-4 k}}{2}$

The solution for $u(x)$ in this case is

$$
u=c_{1} x^{m_{1}}+c_{2} x^{m_{2}}
$$

Then, the solution for $y(x)$ is given by

$$
y=\frac{1}{g} \frac{d}{d x}\left(\ln \frac{u}{f}\right)=-\frac{1}{\alpha} \frac{d}{d x}\left(\ln \frac{c_{1} x^{m_{1}+c_{2} x^{m_{2}}}}{c x^{-\beta / 2}}\right)
$$

or

$$
y=-\frac{1}{\alpha x}\left(\frac{c_{1} m_{1} x^{m_{1}}+c_{2} m_{2} x^{m_{2}}}{c_{1} x^{m_{1}}+c_{2} x^{m_{2}}}+\frac{\beta}{2}\right)
$$

or

$$
y=-\frac{1}{\alpha x}\left(\frac{m_{1} x^{m_{1}}+\bar{c} m_{2} x^{m_{2}}}{x^{m_{1}+\bar{c} x^{m_{2}}}}+\frac{\beta}{2}\right) \quad \bar{c}=c_{2} / c_{1}
$$

Case b: If $(1-4 k)=0 m_{1}=m_{2}=m$ and $m \in \mathbb{R}$. The repeated root is $m=\frac{1}{2}$

The solution for $u(x)$ is

$$
u=c_{1} x^{m}+c_{2} x^{m} \ln x
$$

The solution in this case is obtained as

$$
y=\frac{1}{g} \frac{d}{d x}\left(\ln \frac{u}{f}\right)=-\frac{1}{\alpha} \frac{d}{d x}\left(\ln \frac{c_{1} x^{m}+c_{2} x^{m} \ln x}{c x^{-\beta / 2}}\right)
$$

or

$$
y=-\frac{1}{\alpha}\left(\frac{c_{1} m x^{m-1}+c_{2} x^{m-1}(1+m \ln x)}{c_{1} x^{m}+c_{2} x^{m} \ln x}+\frac{\beta}{2 x}\right)
$$

or

$$
y=-\frac{1}{2 \alpha x}\left(1+\frac{2}{\bar{c}+\ln x}+\beta\right) m=1 / 2 \text { and } \bar{c}=c_{1} / c_{2}
$$

or

$$
y=-\frac{\beta+1}{2 \alpha x}-\frac{1}{x(\alpha \bar{c}+\alpha \ln x)}
$$

Please note that Eq. (84) is equal to Mortici's solution [6] in this case.

Case c: $m_{1}, m_{2} \notin \mathbb{R}, m_{1}=a+b i$ and $m_{2}=a-b i$, where $i=\sqrt{-1}$. The solution for $u(x)$ is

$$
u=e^{-a x}\left(c_{1} \cos b x+c_{2} \sin b x\right)
$$

Hence, the solution for $y(x)$ is finally obtained as

$$
y=\frac{1}{g} \frac{d}{d x}\left(\ln \frac{u}{f}\right)=-\frac{1}{\alpha} \frac{d}{d x}\left(\ln \frac{e^{-a x}\left(c_{1} \cos b x+c_{2} \sin b x\right)}{c x^{-\beta / 2}}\right)
$$

or

$$
y=-\frac{1}{\alpha}\left(\frac{(b \bar{c}-a) \cos b x-(a \bar{c}+b) \sin b x}{\cos b x+\bar{c} \sin b x}+\frac{\beta}{2 x}\right), \bar{c}=c_{2} / c_{1}
$$

\section{Results and Discussion}

In the present work, we have proposed a new transformation which reduces Riccati equation of general type into a second order linear homogeneous differential equation which is readily solvable. The reduced equation has the simplest form of second order. Therefore, there is no need for extra operation to reduce the transformed equation. 
Looking at the form of $g S$, we can immediately see whether the solution is expressed in explicit or implicit form. Since we don't need any proper solution already required for the analytical solution, the present method is further simple and can be preferred in teaching the solutions of Riccati differential equation. Since the method doesn't put any condition on the functions involved in the equation, the method is very general.

According to the present method, proper solutions of Riccati equation can also be obtained. Thus, using the conventional method of transforming the original equation into a linear first order differential equation can be followed. This situation is exemplified in Example 6.

\section{REFERENCES}

[1] Allen, J.L. and Stein, E.M, On the Solution of Certain Riccati Equations, the American Math.Montly, U.S.A., pp.1113-1115, 1964.

[2] Harko, T., Lobo, F.S.N., and Mak, M.K., Analytical Solution of the Riccati Equation with Coefficients Satisfying Integral or Differential Conditions with Arbitrary Functions, Universal Journal of Applied Mathematics, Vol.2, U.S.A., pp.109-118, 2014.
[3] Ince, E.L., Ordinary Differential Equation, ISBN: 978-0-486-60349-0, Dover Publications, New York-U.S.A., pp. 1-204, 1956.

[4] Kreyszig, E., Advanced Engineering Mathematics, ISBN: 0-471-33328-X, John Wiley\&Sons. Inc, New York-U.S.A., pp.1-146, 1999 .

[5] Mak, M.K. and Harko, T., New Integrability Case for the Riccati Equation, Applied Mathematics and Computation, Vol.218, Netherlands, pp.10974-10981, 2012.

[6] Mortici, C., The method of the variation of constants for Riccati Equations, General Mathematics Vol. 16, No. 1, Romania, pp.111-116, 2008)

[7] Pala, Y., Modern Differential Equations and Its Applications, ISBN: 075-591-936-8, Nobel Publications, Bursa-Turkey, pp.1-188, 2006.

[8] Rao, P.R.P., The Riccati Differential Equation, The American Mathematical Montly, U.S.A., pp.995-996, 1962.

[9] Rao, P.R.P. and Ukidave, V.H., Separable forms of the Riccati Equation, The American Mathematical Montly, Vol.75, U.S.A., pp.38-39, 1968.

[10] Siller, H., On the Separability of the Riccati Differential Equation, Mathematics Magazine, Vol.43, No.4, U.S.A., pp.197-202, 1970.

[11] Sugai, I., Riccati's Nonlinear Differential Equation, The American Mathematical Monthly, Vol.67, No.2, U.S.A., pp.134-139, 1960. 